\title{
Exponentially Changeable Quantities; An Attempt to Extend the Transition Time
}

\author{
Constantine Xaplanteris ${ }^{1}$, Loukas Xaplanteris ${ }^{2}$ \\ ${ }^{1}$ Institute of Nanoscience and Nanotechnology (I.N.N.), National Centre for Scientific Research, Athens, Greece \\ ${ }^{2}$ School of Sciences, National University of Athens, Athens, Greece
}

Email address:

cxaplanteris@yahoo.com (C. Xaplanteris)

To cite this article:

Constantine Xaplanteris, Loukas Xaplanteris. Exponentially Changeable Quantities. An Attempt to Extend the Transition Time. International Journal of Biochemistry, Biophysics \& Molecular Biology. Vol. 4, No. 2, 2019, pp. 19-24. doi: 10.11648/j.ijbbmb.20190402.11

Received: February 14, 2019; Accepted: March 25, 2019; Published: October 23, 2019

\begin{abstract}
As many physical changes and conversions are done by exponential mathematical forms during the time that concerns us, the problem rises when the phenomenon has finished, the conversion is completed and the saturation has come upon the changed quantity. Thus, after the saturation is obtained, time becomes unable to provide us with further information and data. The difficulty becomes substantial when those exponential chronicle changes are used on the chronologies and dating of materials which are under scrutiny. Especially when the duration of time is not extended, the results are limited. Those exponential conversions appear in Plasma Physics in the growth or the damping of the plasma waves, as well. With the present theoretical work a non constant coefficient of the conversion is suggested, whose result is the extension of the conversion time. Also, it is proved that the under-duplication time becomes much more extended than it was with the constant conversion coefficient. Furthermore, it is proved that the under-duplication time continually increases as the under-duplications are multiplied. It should be considered that the initial formulation of the basic physical laws (Coulomb law, Biot-Savart law, law of Universal Gravitation, e.t.c) has been done with the first order approach, taking the ratio coefficients as constants. The present study is an extension of the formulation of the well-known laws with the second order approach.
\end{abstract}

Keywords: Exponential Forms, Chronology, Dating of Materials, Semi-life Time, Extension Time

\section{Introduction}

With the present work an effort have been made to penetrate the until now known physical laws, by disputing on the stability of the different "constants" which are implicated to them. So, although the used example in the article is taked from Nuclear Physics, the authors have experience on the plasma waves and thermo- and opto-luminescence dating.

As is well known that when a system is passing from one steady state to another one, it mediates the transitive state, known as transit. The mathematical elaboration during the transitive state is hard enough, and it always has exponential dependence on time. Similar exponential dependence on time is met in mechanical as well as electrical phenomena, such as the re-establishment or interruption of the electrical current. The whole operation of the chrono-circuits is described with exponential functions of time as well.

Another field where exponential forms are met is Plasma Physics, where in the plasma body different waves are developed or extinguished. There, the growth rate or the damping of the waves is described with exponential functions of time [1-6]. Electrical [7-9] and drift [10-12] waves consist obstacles in the thermonuclear process, that is why their study was necessary and has been done thoroughly. Lots of research and many studies on the plasma waves have been carried out at the Plasma Laboratory of 'Demokritos' and many articles and communications have been published already [13-16].

Another instance where exponential functions of time appear is the thermo- and opto-luminescence dating of environmental materials. Much experience on the luminescence dating has been obtained at our laboratory as well [17-20]. So, using the luminescence which is trapped on the material and studying the exponential curve we are able to determine the age when the material was deposited. In Figure 1 the used apparatus is presented (photo).

However, as the transitive phenomenon of the luminescence proceeds to the saturation, the time for this completion is limited and at many times insufficient to reveal 
an extensive dating. Thus, the need for extension of this time led us to seek again another mathematical model, in order to interpret more correctly the exponential phenomena by extending their time [21-23]. Especially, a non constant coefficient of the conversion was taken along with two other constants, which can be found if experimental simulations are used. In this way the extension of the time permits the searching of wider dating.

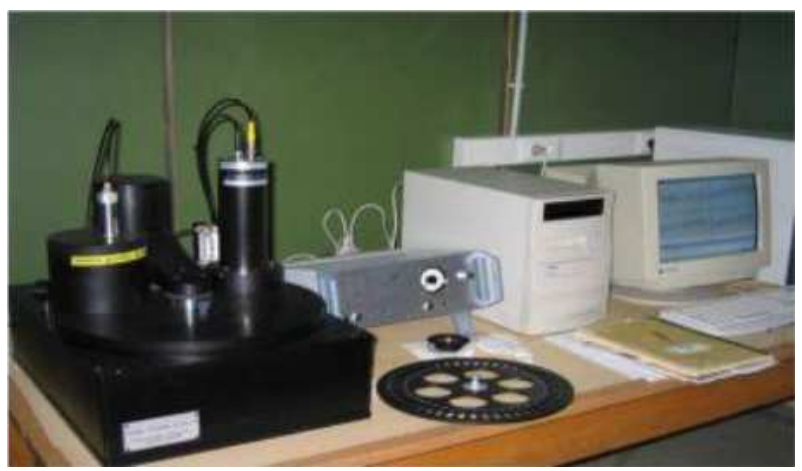

Figure 1. (photo). TL / OSL RISØ-DA-15 reader at the Laboratory of Plasma at NCSR "Demokritos".

The paper is written as following: in Chapter 2 the problem's situation is given. There, the problem is stated both with constant and non constant coefficient $\lambda$. In the next Chapter 3 the study of the law with the non constant coefficient has been done. In Chapter 4 a further study of the above law takes place, whereas in the following Chapter 5 the maximum value for the transition time is given. In the final Chapter 6 the Conclusion and Comments are presented. In Appendix A and B the mathematical elaboration has been done.

\section{The Problem's Situation}

i) As it is well known, according to the radioactivity law, the breaking off coefficient $\lambda$ is taken as constant and we obtain the common result,

$$
N=N_{0} \cdot e^{-\lambda t}
$$

where $N_{0}$ and $N$ are the initial and the unbroken nucleuses respectively, and $\lambda$ the constant coefficient.

From the above law is calculated the semi-life time $t_{1 / 2}$, equal to

$$
t_{1 / 2}=\frac{\ln 2}{\lambda}=\text { const }
$$

It must be noted that for $v=2^{\kappa}$ under-duplications $(\kappa$ is a natural number) the needed time is,

$$
t_{1 / 2^{\kappa}}=\kappa \cdot \frac{\ln 2}{\lambda}
$$

ii) With the consideration below, that $\lambda$ is changing by the time $t$ as,

$$
\lambda=\lambda_{0}-\mu . t
$$

where $\lambda_{0}$ and $\mu$ are constants, then we have the differential equation

below,

$$
d N=-\left(\lambda_{0}-\mu t\right) \cdot N \cdot d t
$$

Eq (5) is solved in Appendix A and its solution is given below,

$$
N=N_{o} \cdot e^{-\lambda_{0} t+\frac{\mu}{2} t^{2}}
$$

\section{The Law (6) study}

i) the semi-life time: by putting $N=\frac{N_{0}}{2}$ into Eq (6) the semi-life time $t_{1 / 2}$ is given with the next equation,

$$
\lambda_{0} t_{1 / 2}-\frac{\mu}{2} t_{1 / 2}^{2}=\ln 2 \text { or } \mu t_{1 / 2}^{2}-2 \lambda_{0} t_{1 / 2}+2 \ln 2=0
$$

That is,

$$
t_{1 / 2}=\frac{\lambda_{0} \pm \sqrt{\lambda_{0}^{2}-2 \mu \ln 2}}{\mu}
$$

ii) the time for $N_{0} / v$ unbroken nucleuses:

if the unbroken nucleuses are $N_{0} / v$, then the required time is $t_{1 / v}$

This time is given with the equation below,

$$
\mu t_{1 / v}^{2}-2 \lambda_{0} t_{1 / v}+2 \ln v=0
$$

and it has the form,

$$
t_{1 / v}=\frac{\lambda_{0} \pm \sqrt{\lambda_{0}^{2}-2 \mu \ln v}}{\mu}
$$

From Eq.s (8) and (10), the solutions with the symbol (+) are given as abnormal results, therefore we consider the solutions only,

$$
\begin{aligned}
& t_{1 / 2}=\frac{\lambda_{0}-\sqrt{\lambda_{0}^{2}-2 \mu \ln 2}}{\mu} \\
& t_{1 / v}=\frac{\lambda_{0}-\sqrt{\lambda_{0}^{2}-2 \mu \ln v}}{\mu}
\end{aligned}
$$

the designation of constants $\lambda_{0}$ and $\mu$ 
By using the time $t_{1 / v}$ from Eq.(10), which is required for the unbroken nucleuses diminished at $N=N_{0} / v$ value, for two measurings $v_{1}$ and $v_{2}$ and by putting, $t_{1 / v_{1}}=\alpha$ and $t_{1 / v_{2}}=\beta$, then the Eq.(10) gives the system,

$$
\begin{aligned}
& \frac{\lambda_{0}-\sqrt{\lambda_{0}^{2}-2 \mu \ln v_{1}}}{\mu}=\alpha \\
& \frac{\lambda_{0}-\sqrt{\lambda_{0}^{2}-2 \mu \ln v_{2}}}{\mu}=\beta
\end{aligned}
$$

The experimental values $\alpha$ and $\beta$ can be found if simulations are used in the experiment.

The system's solution gives the value of the constants $\lambda_{0}$ and $\mu$, as following,

$$
\lambda_{0}=\frac{\beta^{2} \ln v_{1}-\alpha^{2} \ln v_{2}}{\alpha \beta(\beta-\alpha)}
$$

and

$$
\mu=\frac{2\left(\beta \ln v_{1}-\alpha \ln v_{2}\right)}{\alpha \beta(\beta-\alpha)}
$$

Application:

By taking the lessenings, for $v_{1}=4$ and $v_{2}=8$, then the relations of Eq.s (12) give,

$$
\lambda_{0}=\frac{\left(2 \beta^{2}-3 \alpha^{2}\right) \cdot \ln 2}{\alpha \beta(\beta-\alpha)} \text { and } \mu=\frac{2(2 \beta-3 \alpha) \cdot \ln 2}{\alpha \beta(\beta-\alpha)}
$$

iii) the time extension:

Let us consider that $N_{0}$ originally unbroken nucleuses will be subject to $v=2^{\kappa}$ under-duplications ( $\kappa$ is a natural number). The needed time for this conversion $t_{1} / v$ will be calculated in two ways: $\alpha$ ) with the consideration of the constant coefficient $\lambda_{0}$, when it is $t_{1 / v}=\kappa \frac{\ln 2}{\lambda_{0}}$, and b) with the non constant coefficient $\lambda=\lambda_{0}-\mu t$, when it is

$$
t_{1 / v}=\frac{\lambda_{0}-\sqrt{\lambda_{0}^{2}-2 \mu \ln v}}{\mu} \text { or } t_{1 / v}=\frac{\lambda_{0}-\sqrt{\lambda_{0}^{2}-2 \mu \kappa \ln 2}}{\mu}
$$

In Appendix B the mathematical elaboration is given, which has proved that

$$
\frac{\lambda_{0}-\sqrt{\lambda_{0}^{2}-2 \mu \kappa \ln 2}}{\mu} \succ \kappa \frac{\ln 2}{\lambda_{0}}
$$

\section{Further Study of the Eq.(6)}

i) the Eq.(6) study: The Eq.(6) my be written as,

$$
N=\frac{N_{0}}{e^{-\frac{\mu}{2} t^{2}+\lambda_{0} t}}
$$

The exhibitor of the denominator is written as,

$$
\psi=-\frac{\mu}{2} t^{2}+\lambda_{0} t
$$

and its behavior-study is shown in Figure 2.

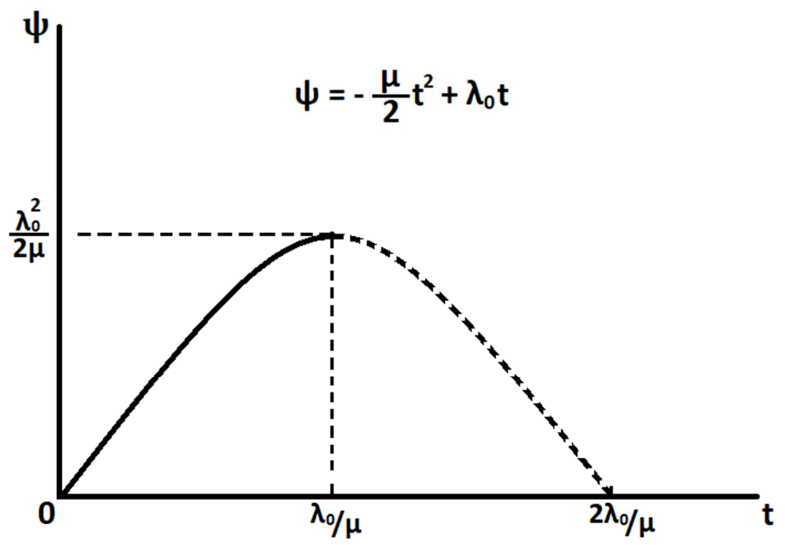

Figure 2. The exhibitor of the denominator vs timet .

From Figure 2 it must be noticed that $\psi=0$, for $t=0$ and $t=\frac{2 \lambda_{0}}{\mu}$.

A maximum appears at the time $t=\frac{\lambda_{0}}{\mu}$. The minimum's value is,

$$
\psi_{\max }=\frac{\lambda_{0}^{2}}{2 \mu}
$$

In Figure 3 the relation $N=N(t)$ is presented, as written in Eq. (6)

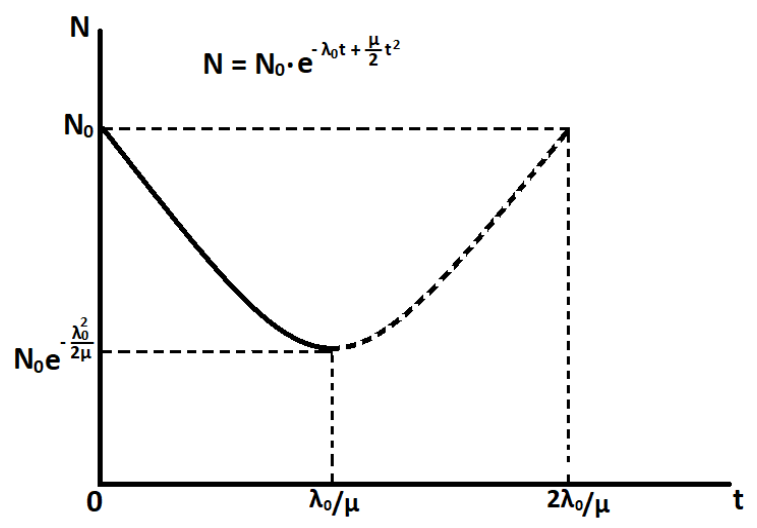

Figure 3. The unbroken nucleuses vs time $t$. 
From Figure 3 it must be noticed that the actual time for the realization of the phenomenon is $t=\frac{\lambda_{0}}{\mu}$, where the $N$ is diminished, until it reaches the minimum value, $N_{0} \cdot e^{-\frac{\lambda_{0}^{2}}{2 \mu}}$

ii) the Eq.(14) study:

The Eq. (14) can be written as

$$
t_{1 / v}=\frac{\lambda_{0}}{\mu}-\sqrt{\left(\frac{\lambda_{0}}{\mu}\right)^{2}-\frac{2 \kappa}{\mu} \cdot \ln 2}
$$

and by putting $\frac{\lambda_{0}}{\mu}=a$ and $\frac{2 \ln 2}{\mu}=b$ it results to

$$
t_{1 / v}=a-\sqrt{(a)^{2}-b \cdot \kappa} \text { or } \sqrt{a^{2}-b \cdot \kappa}=a-t_{1 / v}
$$

Finally, this gives the following,

$$
t_{1 / v}^{2}-2 a t_{1 / v}+b \kappa=0
$$

Eq. (20) has the solution,

$$
t_{1 / v}=\alpha \pm \sqrt{\alpha^{2}-b \kappa}
$$

Three points must be isolated and are tabulated in Table 1 below:

Table 1. Three distinct points which describe the function of the Eq. (21).

\begin{tabular}{ll}
\hline The factor of the under-dublications $b \kappa$ & The needed time $t_{1 / v}$ \\
\hline 0 & 0 \\
0 & $2 \alpha$ \\
$\alpha^{2}$ & $\alpha$ \\
\hline
\end{tabular}

The study of the last Eq. (21) is shown in Figure 4, where the time $t_{1 / v}$ is presented versus the quantity $b \kappa$.

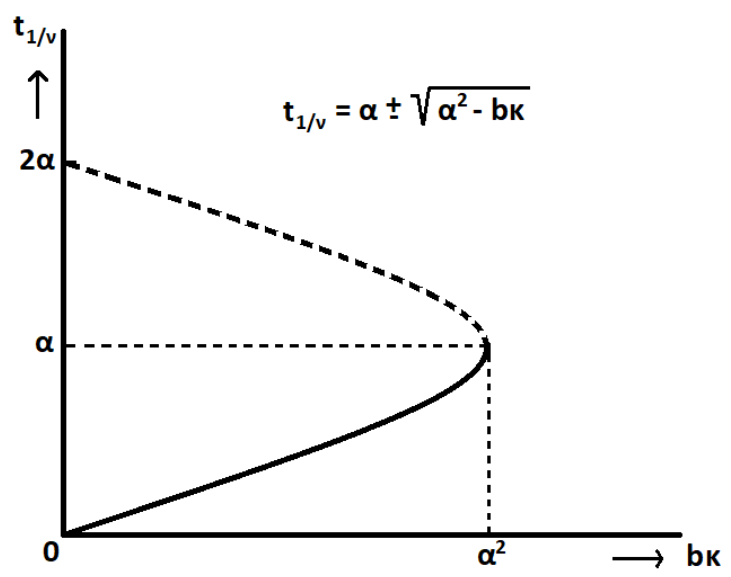

Figure 4. The time $t_{1 / v}$ is presented vs the quantity $b \kappa$.
The solid curve describes the real phenomenon.

\section{The Maximum Value for the Transition Time}

The transition time $t_{\max }$ can be found in two ways: i) by annihilating the constant $\lambda=0$, from which we take $t_{\max }=\lambda_{0} / \mu$, or ii) from the time subdivision $t_{1 / v}$ when annihilating the quantity $\lambda_{0}^{2}-2 \mu \ln v=0$, where we can again find that,

$$
t_{1 / v}=\frac{\lambda_{0}-\sqrt{\lambda_{0}^{2}-2 \mu \ln v}}{\mu}=\frac{\lambda_{0}-\sqrt{0}}{\mu} \Rightarrow t_{1 / v}=t_{\max }=\frac{\lambda_{0}}{\mu}
$$

If we consider the subdivision $v$ as power of the 2 , $v=2^{\kappa}$ with $\kappa$ as a natural number, then we calculate the number $\kappa$ of sub duplications, as

$$
\Rightarrow \lambda_{0}^{2}-2 \mu \ln v=0 \Rightarrow \lambda_{0}^{2}-2 \mu \ln 2^{\kappa}=0 \quad \Rightarrow \quad \lambda_{0}^{2}-2 \kappa \mu \ln 2=0
$$

$$
\kappa=\frac{\lambda_{0}^{2}}{2 \mu \ln 2}
$$

\section{Conclusion-Comments}

Taking into consideration that the coefficient $\lambda$ is non constant, and it is changeable by time, many advantages can be seen with the problem's solution:

i) the semi-duplication time $t_{1 / 2}$ is not constant, but it increases as the unbroken nucleuses decrease.

ii) The originally unbroken nucleuses $N_{0}$, are not broken as a whole, but the quantity $N_{0} \cdot e^{-\lambda_{0}^{2} / 2 \mu}$ remains unbroken.

iii) As the proposed problem and its solution is generally one and it includes the known problem with $\lambda=$ const, it is enough to put $\mu=0$ in the results.

iv) The proposed mathematical form for the coefficient $\lambda$ is linear and the elaboration is relatively simple, although it has more texture than the case with $\lambda=$ const .

v) The conversion's coefficient $\lambda$ appears, which has the constants $\lambda_{0}$ and $\mu$ that can be found experimentally.

With the present study we once again seek more information and data for the quantities which are changeable by time, so that to extend the time of the materials dating problem. In this way, we must not be sufficed with a simplification of the problem by the consideration that $\lambda$ is constant. On the contrary, it must be considered that $\lambda$ is non constant and it has chronicle dependences through which we get much more results. 
Another idea is to seek successive conversions of the materials, so that, each time, the conversion time is added to a continuous time.

\section{Acknowledgements}

The authors would like to thank researcher Eystathios Sideris for his collaboration on Plasma Physics in the past, as well as the technical staff of the plasma laboratory of the reshearch center 'Demokritos' for their help during the experiments. Also, we would like to thank researchers Eyaggelos Tsakalos and Maria Kazantzaki for their guidance on the thermo- and opto-luminescence dating of objects. Furthermore, we thank Dr Jenny Vitopoulou for proof reading and helping with the preparation of the manuscript.

\section{Appendix}

\section{Appendix $A$}

The Eq (5) may be written as,

$$
\frac{d N}{N}=-\left(\lambda_{0}-\mu t\right) \cdot d t
$$

and after its intergration the next equation is resulted,

$$
\ln N=-\lambda_{0} t+\frac{\mu}{2} t^{2}+C
$$

where $C$ is constant defined from the initial conditions: for time $t=0$, is valid that $N=N_{0}$. So, is $C=\ln N_{0}$ and the solution resulted at Eq (6)

$$
N=N_{o} . e^{-\lambda_{0} t+\frac{\mu}{2} t^{2}}
$$

\section{Appendix B}

This mathematical proof takes place by using the mathematical inducement method. So, it is considered that is valid the inequality below,

$$
\frac{\lambda_{0}-\sqrt{\lambda_{0}^{2}-2 \mu \kappa \ln 2}}{\mu} \succ \kappa \frac{\ln 2}{\lambda_{0}}
$$

Then it can be written as, $\sqrt{\lambda_{0}^{2}-2 \mu \kappa \ln 2} \prec \lambda_{0}-\frac{\kappa \mu}{\lambda_{0}} \ln 2$ or

$$
\lambda_{0}^{2}-2 \mu \kappa \ln 2 \prec \lambda_{0}^{2}-2 \mu \kappa \ln 2+\left(\frac{\mu \kappa}{\lambda_{0}} \ln 2\right)^{2}
$$

From which it is resulted that

$$
0 \prec\left(\frac{\mu \kappa}{\lambda_{0}} \ln 2\right)^{2}
$$

which is valid.

\section{References}

[1] Block, D., Piel, A., Schroder, Ch. and Klinger, T. 2001, Syncronization of drift waves. Phys. Rev. E 63, 056401.

[2] Chen, F. 1964 Normal Modes for Electrostatic Ion Waves in an Inhomogeneous Plasma. Phys. Fluids 7, pp. 949-955.

[3] D' Angelo, N. and Motley, R. 1963 Low Frequency Oscillations in a Potassium Plasma. Phys. Fluids 6 (3), pp. 422-425.

[4] L. Spitzer, 1967, Physics of Fully Ionized Gases, $2^{\text {nd }}$ edn. New York: John Wiley \& Sons.

[5] H. W. Hendel, B. Coppi F. Perkins and P. A. Politzer, 1967, Collisional Effects in Plasmas-Drift- Wave Experiments and Interpretation, Phys. Rev. Lett. 18. 439).

[6] Ellis, R., Marden-Marshall, E and Majeski, R. 1980 Collisional drift instability of a weekly ionized argon plasma. Plasma Phys. 22, pp. 113-132.

[7] D L. Tang, A P Sun, X M Qiu, and Paul K Chu, "Interaction of Electromagnetic Waves with a Magnetized Nonuniform Plasma Slab”, IEEE Transactions On Plasma Science, VOL. 31, NO. 3, 2003.

[8] C S Gurel and E Oncu, "Interaction of Electromagnetic Wave and Plasma Slab with Partially Linear and Sinusoidal Electron Density Profile", Progress In Electromagnetics Research Letters, Vol. 12, 171-181, 2009.

[9] A Yeşil and İ Ünal, "Electromagnetic Wave Propagation in Ionospheric Plasma”, In Tech Europe, Vol 10, pp. 190-212, 2011.

[10] Hendel, H. W., Coppi, B., Perkins, F. and Politzer, P. A. 1967. Collisional effects in plasmas-drift-wave experiments and interpretation. Phys. Rev. Lett. 18 pp. 439-442.

[11] Marden-Marshall, E., Ellis, R. F. and Walsh, J. E. 1986 Collisional drift instability in a variable radial electric field. Plasma Phys. 28 (9B), pp. 1461-1482.

[12] A. J. Anastassiades and C. L. Xaplanteris. Growth of a drift wave due to an RF-Field in a magnetized plasma. Bulletin of the American Physical Society, Vol. 25, Issue 8, page 866, 1980.

[13] A. J. Anastassiades and C. L. Xaplanteris. Drift Wave Instability in the presence of an RF-Field in Magnetized Plasma. Journal of the Physical Society of Japan V/52, p-p 492-500, Febr. 1983.

[14] C. L. Xaplanteris. Effect of Low Frequency Instability on Hall Conductivity in Plasma. Astrophysics and Space Science Vol 139 number 2, p-p 233-242, December 1987.

[15] C. L. Xaplanteris. Collisional instability into a rare magnetized plasma. An experimental model for magnetospheric and space plasma study. Journal of Plasma Physics Vol 75 Issue 03 pp. 395-406, June 2009.

[16] Tsakalos, E., Christodoulakis, J., Charalambous, L., 2016. Dose Rate calculator (DRc) - A Java application for dose rate and age determination based on luminescence and ESR dating. Archaeometry, DOI: 10.1111/arcm.12162.

[17] Kazantzaki, M., Athanassas, C., Bassiakos, Y., Tsakalos E., 2016. Luminescence dating of Quaternary Coastal deposits of Evoikos Gulf (central Greece). British Archaeological Reports. Chapter 30 published in BAR S2780 Proceedings of the 6th Symposium of the Hellenic Society for Archaeometry, 207-215. 
24 Constantine Xaplanteris and Loukas Xaplanteris: Exponentially Changeable Quantities. An Attempt to Extend the Transition Time

[18] Tsakalos, E., Athanassas, C., Tsipas, P., Triantaphyllou, M., Geraga, M., Papatheodorou, G., Filippaki E., Christodoulakis, J., Kazantzaki, M., 2016. Luminescence geochronology and paleoenvironmental implications of coastal deposits of southeast Cyprus. Journal of Archaeological and Antropological Sciences, DOI: 10.1007/s12520-016-0339-7.

[19] Tsakalos, E., Dimitriou, E., Kazantzaki, M., Anagnostou Ch., Christodoulakis J., Filippaki E., 2018. Testing optically stimulated luminescence dating on sand-sized quartz of deltaic deposits from Sperchios delta plain, Greece. Journal of Palaeogeography, DOI: 10.1016/j.jop.2018.01.001.

[20] C. L. Xaplanteris, L. C. Xaplanteris and D. P. Leousis.
Mathematical Modeling on the Exponential Changed Plasma Quantities leads to the more Persuasive Answers. Chaotic Modeling and Simulation (CMSIM) Vol 1: pp 109-128, 2014.

[21] C. L. Xaplanteris, L. C. Xaplanteris and D. P. Leousis. An attempt to Study the Growth rate and Damping Profoundly. Approximate solutions by using Mathematical Models. Plasma Science and Technology Vol. 16. No. 10. pp, 897-906, Oct. 2014.

[22] C. L. Xaplanteris, L. C. Xaplanteris and D. P. Leousis, Approximate models for the study of exponential changed quantities: Application on the plasma waves growth rate or damping. AIP ADVANCES 4, 037123 (2014). 